THE KURUME MEDICAL JOURNAL

Vol.27, p. 263-268, 1980

\title{
PRIMITIVE TRIGEMINAL ARTERY AND INTRACRANIAL CAROTID OCCLUSION
}

\author{
- A CASE PEPORT-
}

\author{
MINORU SHIGEMORI ${ }^{1 *}$, MORIHISA SHIRAHAMA ${ }^{1)}$, FUMIHITO YAMAMOTO ${ }^{2)}$, \\ KUNITADA HARA ${ }^{1)}$, TAKASHI TOKUTOMI ${ }^{2)}$ AND OSAMU NAKASHIMA ${ }^{2)}$ \\ Division of Neurosurgery, Omuta City Hospital, Omuta, 8361), \\ Department of Neurosurgery, Kurume University School of \\ Medicine, Kurume, 8302), Japan
}

Received for publication October 4, 1980

\begin{abstract}
A patient with a primitive trigeminal artery who presented with homolateral intracranial carotid occlusion following transient ischemic attacks (TIA's) is described. The clinical significance of the primitive trigeminal artery in patients with occlusive cerebrovascular disease is discussed.
\end{abstract}

\section{INTRODUCTION}

A primitive trigeminal artery is known to be associated with other intracranial lesions. It is still unknown however whether the primitive trigeminal artery has a significant role in patients with occlusive cerebrovascular disease. This report describes a case with primitive trigeminal artery which presented with homolateral intracranial carotid occlusion at the cavernous portion of the artery following transient ischemic attacks (TIA's).

\section{CASE REPORT}

A 73 year-old female with hypertension was well until August 15, 1979, when she complained of a speech disturbance lasting for several hours and was admitted to a local hospital. On
August 25, a transient episode of motor aphasia associated with right hemiparesis was observed and she was transferred to Omuta City Hospital. On admission, she was alert, right spastic hemiparesis and motor aphasia were noted. A general physical examination was within normal limits except for hypertension. Blood pressure was unstable and showed $190-90 \mathrm{mmHg}$ on the right, $186-90 \mathrm{mmHg}$ on the left. There was no history of previous neurological diseases or cardiac diseases. CT scan demonstrated a localized small low density area on the left parietal lobe and mild brain atrophy. Left carotid angiography showed an elongation of the internal carotid artery with a primitive trigeminal artery which originated from the gasserian portion of the carotid artery, through which the rostral part of the basilar artery, left posterior cerebral artery and bila-

* Present address: Minoru Shigemori, M. D.

Division of Neurosurgery, Syakaihoken Tagawa Hospital, 10-18, Kamihonmachi, Tagawa City, Fukuoka. 
teral superior cerebellar arteries were well visualized (Fig. 1). The primitive trigeminal artery was almost the same size as the intracranial carotid artery distal to the primitive artery. Right brachial angiography demonstrated a well visualized right posterior communicating artery and posterior cerebral artery. The right vertebral artery terminated as the right posterior inferior cerebellar artery. Left brachial angiography revealed a normal appearance of the left posterior inferior cerebellar artery and faintly filled caudal basilar artery (Fig. 2). These studies indicated that the left internal carotid artery supplied the rostral brain stem and cerebellum as well as the left cerebral hemisphere. Left cervical carotid angiography demonstrated an elongation and dilatation of the internal carotid artery suggesting

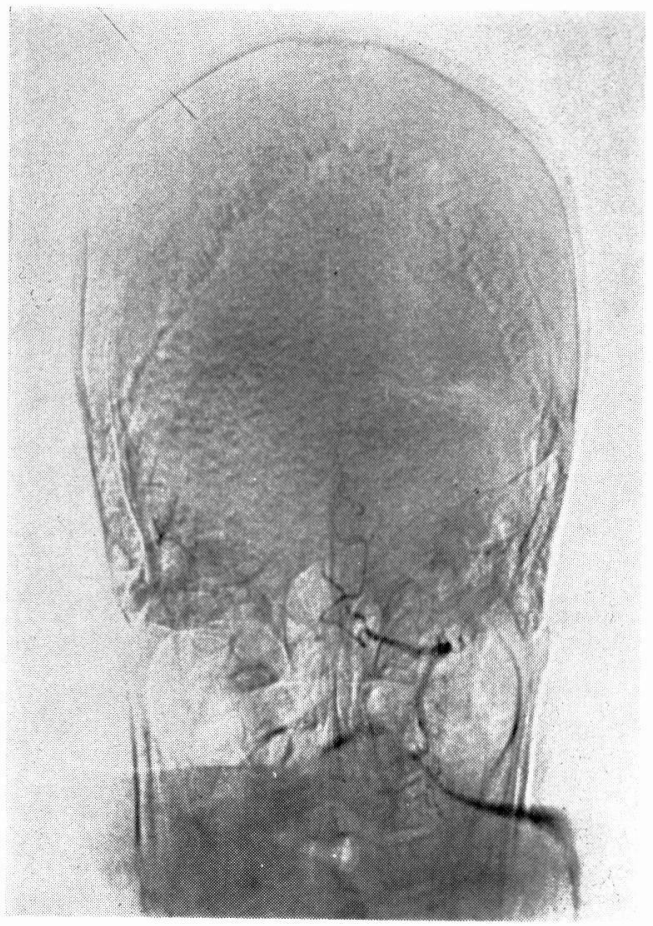

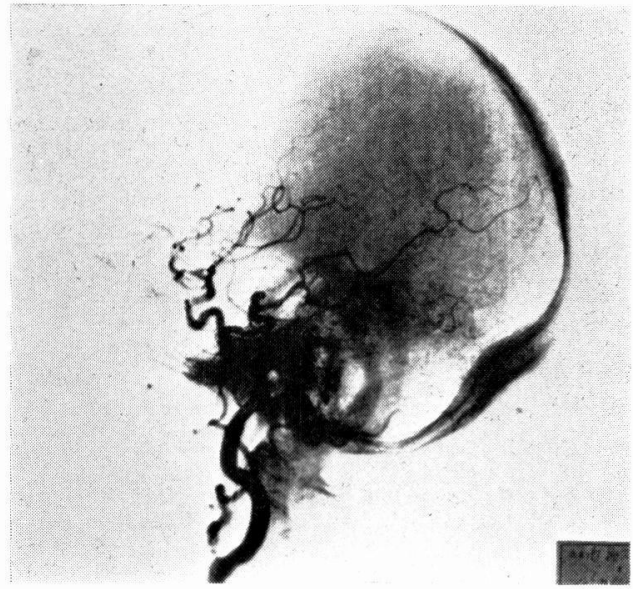

Fig. 1. Left carotoid angiogram on admission shows left primitive trigeminal artery and elongation of the internal carotid artery. The cavernous portion of the carotid artery is almost the same size as the primitive trigeminal artery through which rostral basilar, left posterior cerebral and bilateral superior cerebellar arteries are well demonstrated.

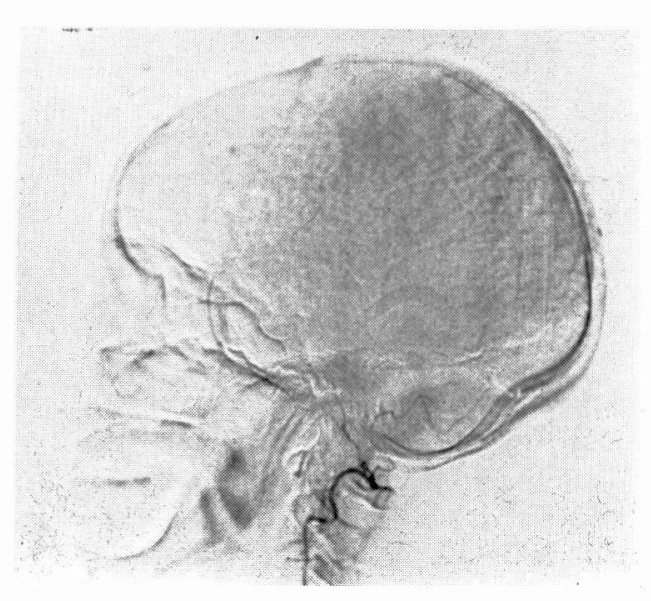

Fig. 2. Left brachial angiogram showing left vertebro-basilar system (A-P and Lateral view). The caudal basilar artery was faintly visalized at the skull base. 
arteriosclerosis (Fig. 3). However there was no ulceration or stenosis of the carotid bifurcation.

The diagnosis of left cerebral stroke was made and medical treatment included antiagglutinating agents and vasodilators. These failed to improve the neurological deficits however, and right hemiparesis as well as motor aphasia persisted. Blood pressure also remained uncontrollable. On September 24, the patient had deteriorated and became semicomatous, exhibiting anisocoria and left conjugate deviation. Emergency CT scanning revealed a diffuse low density area on the left cerebral hemisphere and shift of the midline structure to the right indicating

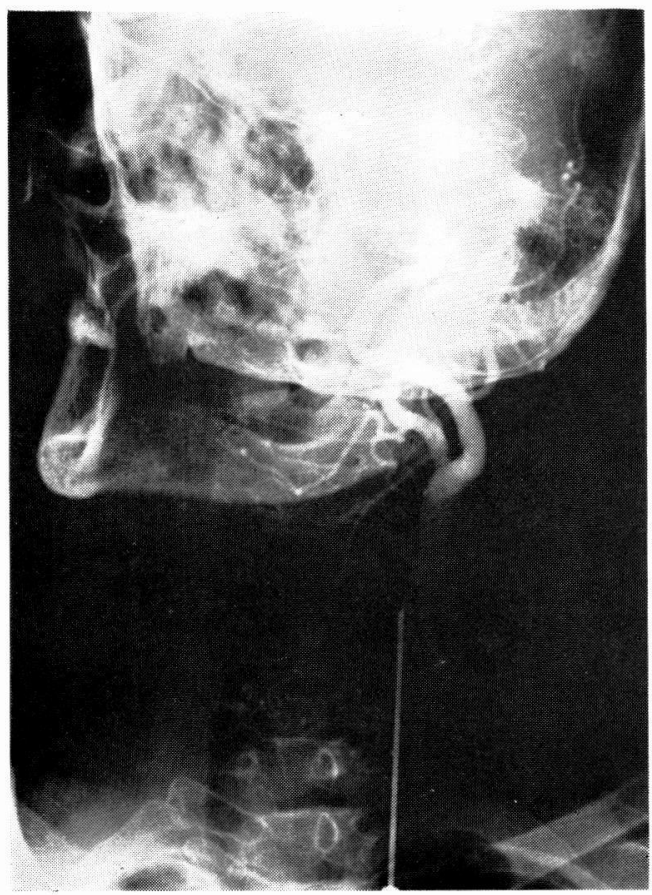

Fig. 3. Left cervical carotid angiogram (Oblique view) indicated the elongation and dilatation of the internal carotid artery and its bifurcation. There are no ulcerations or stenosis. extensive cerebral infarction on the left side (Fig. 4). Left carotid angiography demonstrated an incomplete occlusion of the intracranial carotid artery at the level of the cavernous portion distal to the origin of the primitive trigeminal artery and poorly filled intracranial carotid bifurcation. Dilatation of the branches of the left external carotid artery and retrograde filling of the anterior cerebral circulation through the left posterior cerebrai artery were also observed (Fig. 5). In spite of vigorous medical treatments with corticosteroids and hypertonic solutions, the neurological deficits remain and the patient is severely disabled.

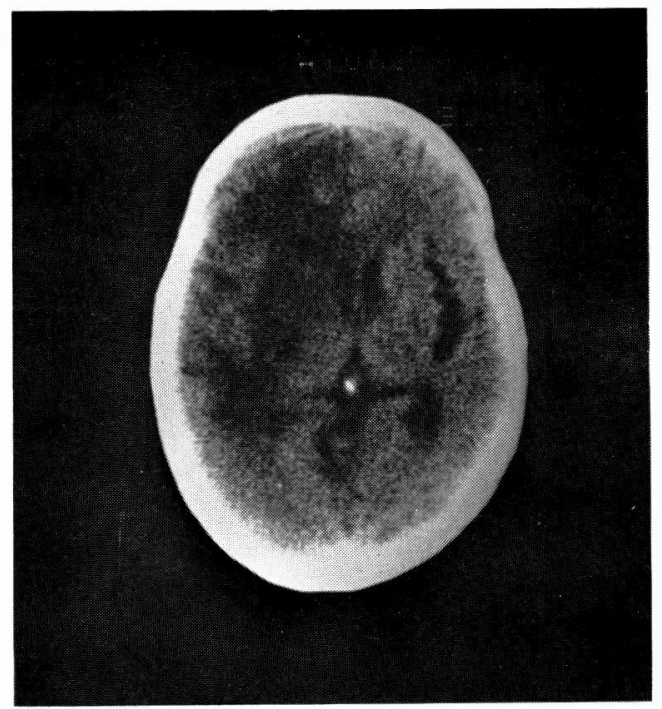

Fig. 4. CT scan detected after major stroke showing diffuse low density areas on the left cerebral hemisphere with mild shift of the midline structure. 

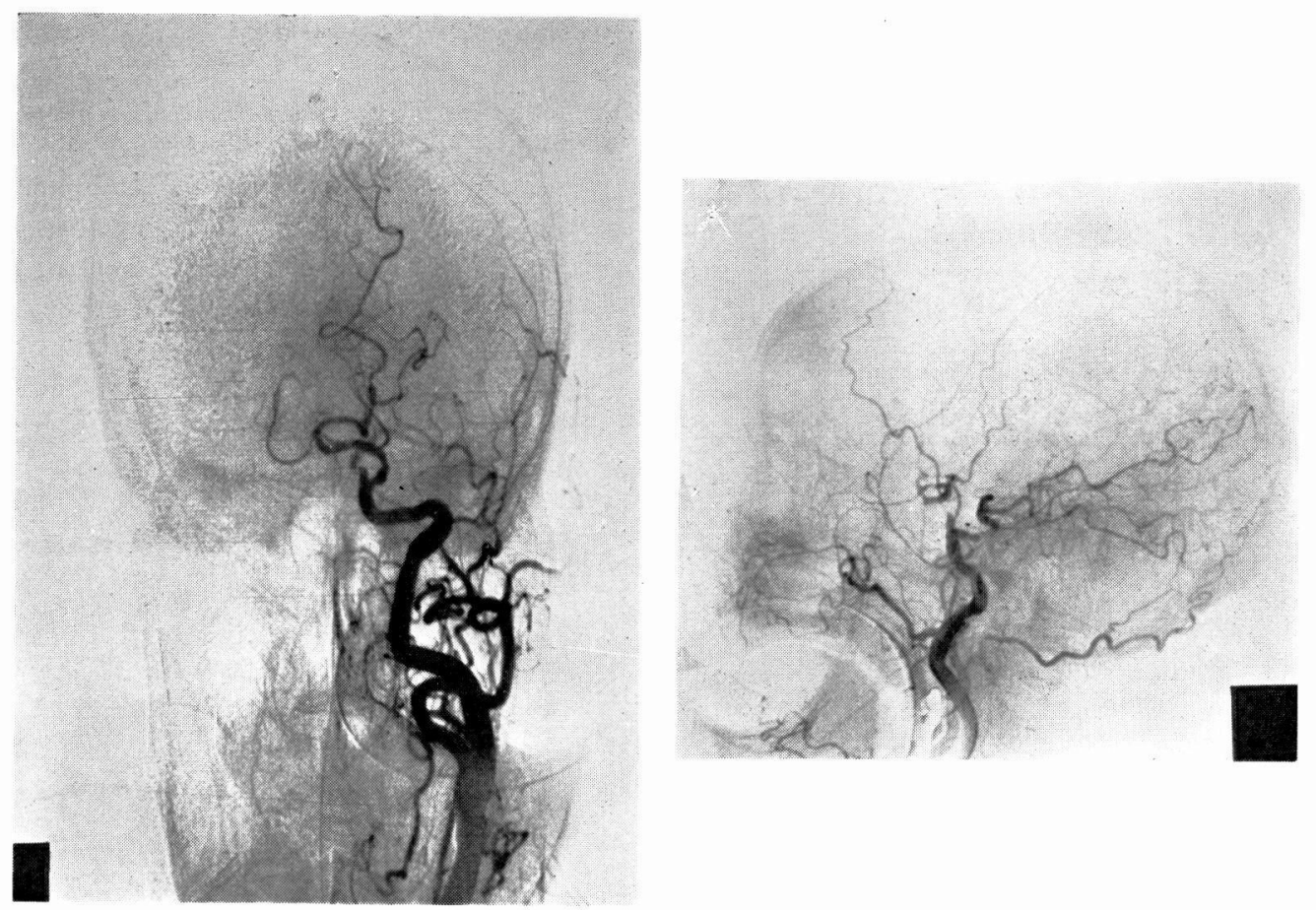

Fig. 5. Left carotid angiogram, A-P (left) and lateral (right) view detected after major stroke showing an occlusion of the intracranial carotid artery at the cavernous portion distal to the origin of the primitive trigeminal artery and faintly opacified intracranial carotid bifurcation.

\section{DISCUSSION}

Angiographic demonstration of the primitive trigeminal artery is most common among the persistent carotidbasilar anastomosis, with an incidence of 0.1 to $0.2 \%$ (Eadie et al., 1964; Lie, 1968). Several authors have reported on the clinical significance of the primitive trigeminal artery, and have suggested cranial nerve compression in the cavernous sinus (Kempe and Smith, 1969; Sunderland., 948) or an aneurysm formation (Wolpert, 1966). As possible causes, it has also been suggested that cerebrovascular insufficiency may occur because of alteration of cerebral circulation due to a primitive trigeminal artery (Bingham and Hayes, 1961; Field, 1968; Nielsen and Jonson, 1967). A possible protective role for the trigeminal artery in occlusive cerebrovascular disease has not been well documented (Khodadad, 1977; Stern et al., 1978; Waller et al., 1977).

In the present case which corresponded to the first type classified by Saltzman (1959), the blood supply to the left cerebral hemisphere, rostral brain stem and cerebellum was fed by the left internal carotid artery and the left posterior communicating artery seemed to be hypoplastic. Left carotid angiography showed a moderated narrowing of the cavernous portion of the internal carotid artery distal to the primitive trigeminal artery and there were no ulcerations or stenosis of the left cervical carotid bifurcation. The blood pressure of the patient was un- 
stable and the neurological abnormalities were related to the left cerebral hemisphere rather than to the brain stem. Furthermore, the left cerebral stroke subsequent to TIA's resulted from incomplete occlusion of the intracranial carotid artery at the level of the cavernous portion. These facts suggest that the sequence of events in this patient was probably not embolic in nature and that the completed stroke following TIA's resulted from thrombus formation at the level of the cavernous portion of the left intracranial carotid artery. It is also possible to suppose that the transient ischemic attack of the patient before admission was caused by relatively reduced blood flow to the left cerebral hemisphere due to shunting or "steal" phenomenon through the primitive trigeminal artery. The hemodynamic changes in the intracranial carotid artery might have played some role in the production of thrombus at the cavernous portion where arteriosclerotic narrowing existed.

Thus the presence of the primitive trigeminal artery does not appear to have a major protective role in patients with occlusive cerebrovascular disease as suggested by Khodadad (1977). Rather, the primitive trigeminal artery may play an important role in precipitating cerebral infarcts in some cases. Since this patient's stroke was caused by the reduction of the cerebral blood flow, superficial temporal artery-middle cerebral artery anastomosis (STAMCA anastomosis) may have improved the neurological signs if performed during the period of TIA's. If TIA's involving the brain stem were present in a patient with primitive trigeminal artery and which resulted from microemboli originating in the cervical carotid artery, as noted in the recent reports (Stern et al., 1978; Waller et al., 1977), a carotid endoarterectomy may have provided sufficient relief from symptoms and may have prevented the development of cerebral infarct.

\section{ACKNOWLEDGEMENTS}

The authors gratefully give thanks to prof. Shinken Kuramoto for his advice and review of the manuscript.

\section{REFERENCES}

Bingham, C. W. and Hayes, G. J. (1961). Persistent carotid-basilar anastomosis. J. Neurosurg. 18, 398-400.

EAdie, M. J., Jamieson, K. G. and LenNon, E. A. (1964). Persisting carotid-basilar anastomosis. J. Neurol. Sci. 1, 501-511.

FIELD, W. S. (1968). The significance :of persistent trigeminal artery: Carotid basilar anastomosis. Radiology, 91, 1096-1101.

Kempe, L. G. and Smith, D. R. (1966). Trigeminal neuralgia, facial spasm, intermedius and glossopharyngeal neuralgia with persistent carotid basilar anastomosis. J. Neurosurg. 131, 445-451.

KhodAdAD, G. (1977). Trigeminal artery and occlusive cerebrovascular diseases. Stroke, 8, 177-181.

LIE, T. A. (1968). Congenital anomalies of the carotid arteries; including the carotid-basilar and carotid-vertebral anastomoses on angiographic study and a review of the literature. Amsterdam: Excerpta Medica, 76-84.

Nielsen, P. G. and Jonson, M. (1967). Persistent primitive trigeminal artery demonstrated tby vertebral angiography. Am. J. Roentgenol. 101, 47-51.

Saltzman, G. F. (1959). Patent primitive trigeminal artery studied by cerebral angiography. Acta Radiol. 51, 329-336.

Stern, J., Correll. J. W. and Bryan, N. (1978). Persistent hypoglossal artery and persistent trigeminal artery presenting with posterior fossa transient ischemic attacks. J. Neurosurg. 49, 614-619.

Sunderland, S. (1948). Neurovascular relations and anomalies at the base of the brain. J. Neurol. Neurosurg. Psychiat. 11, 243-257. Waller, F. T., Simons, R. L., Kerber, C., 
Kiesel, I. O. and TAnabe, C. T. (1977). Trigeminal artery and microemboli to the brain stem. J. Neurosurg. 46, 104-106.
Wolpert, S. M. (1966). The trigeminal artery and associated aneurysm. Neurology, 16, 610-614. 